\title{
How Reliable is the Cuprates System to Recent Technology?
}

\author{
Emetere M. E. ${ }^{1}$, Awojoyogbe O. B. ${ }^{2}$, Uno U. E. ${ }^{2}$, Isah K. U. ${ }^{2}$, Sanni E. S. ${ }^{3}$, \& Akinyemi M. L. ${ }^{1}$ \\ ${ }^{1}$ Department of Physics, Covenant University, Canaan land, P.M.B 1023, Ota-Nigeria \\ ${ }^{2}$ Department of Physics, Federal University of Technology, Minna-Nigeria \\ ${ }^{3}$ Department of Chemical Engineering, Covenant University, Ota-Nigeria
}

\begin{tabular}{ll}
\hline \hline Article Info & ABSTRACT \\
\cline { 2 - 3 } Article history: & $\begin{array}{l}\text { The emergence of cuprates as a high Tc superconductor gave high hopes in } \\
\text { the discovery of a room temperature superconductor. It is almost three } \\
\text { decades and the highest critical temperature attained on the cuprates is about }\end{array}$ \\
$\begin{array}{l}\text { Received Dec 27, } 2015 \\
\text { Accepted Mar 14, 2016 }\end{array}$ & $\begin{array}{l}\text { 135K. A brief overview was conducted on the progress made so far on the } \\
\text { cuprates. A mathematical approach was used to design a formula which } \\
\text { could determine the experimental results of critical temperature of versed } \\
\text { cuprates superconductors. The result of our findings shows that the } \\
\text { Keyword: }\end{array}$ \\
$\begin{array}{l}\text { possibility of attaining the experimental room temperature cuprates } \\
\text { superconductor seems very narrow. The study recommended an elaborate } \\
\text { approach on the hybridization of cuprates for future research. Hence, there is } \\
\text { puprates }\end{array}$ & $\begin{array}{l}\text { possibility of having cuprates with wide engineering application. } \\
\text { Proton Mass Participation }\end{array}$
\end{tabular}
Hybridization All rights reserved.

\section{Corresponding Author:}

Emetere Moses Eterigho,

Department of Physics,

Covenant University,

Canaan land, P.M.B 1023, Ota-Nigeria.

Email: emetere@yahoo.com

\section{INTRODUCTION}

Since the emergence of the concept of superconductivity by Kamerlingh Onnes in 1911[1], numerous scientific research work were conducted to explain the theory of superconductivity. Joseph John Thompson [2] made a proposal that superconductivity was due to fluctuating electric dipole chains while Einstein [3] explained that the theory of superconductivity was due to molecular conduction chain which was independent on non-interacting electrons in solids. In 1928, after the formulation of quantum mechanics, Heisenberg [4] and Felix Bloch [5] made their contributions by introducing the theory of magnetism and the theory of electrons in crystals respectively. Bloch's second theorem on superconductivity - coupled spontaneous currents and coherent quantum motion of electrons, was proved wrong by David Bohm [6]. Landau [7],[8] proposed the free energy expansion of antiferromagnets and the theory of phase transition. Léon Brillouin [9] supported Bloch's first theorem where he claimed that superconductivity was a metastable state. Walter Meissner and Robert Ochsenfeld demonstrated that the magnetic flux is expelled from a superconductor regardless of its state. This idea was corroborated by the London theory of penetration depth [10]. Werner Heisenberg gave a new dimension to the theory of superconductivity by investigating the bound states of the Fermi energy. This theory was vehemently criticized by Fritz London [10], he proposed that superconductivity was due to vibrations of the crystal lattice which affirmed clearly that a superconductor in a coherent quantum state are macroscopic.

Fröhlich [11] discovered that the vibrations of the crystalline lattice engendered a net attraction between electrons. He suggested that a likely cause of superconductivity. In 1954, Fröhlich proposed the high temperature superconductor. In 1957, Bardeen, Cooper, and Schrieffer [12] came out with the microscopic (BCS) theory - based on conceptual and mathematical foundation for conventional superconductivity. The BCS theory actually explained the mechanism for the convectional superconductivity. Unarguably, one of the 
early initiators of the microscopic treatment of convectional superconductor is the BCS theory. Though its progress was short-lived as other types of high-temperature superconductors emerged. The microscopic treatment of superconductor on its own is a valid theory. However, the acceptance of the microscopic theory did not exempt the validation of the BCS theory. It has been argued that it lacked salient concept of superconductivity [13]. Frönlich's prediction came through in early 1986 when Georg Bednorz and Alex Müller discovered the copper oxides (cuprates) containing lanthanum $(\mathrm{LaBa})_{2} \mathrm{CuO}_{4}$ with critical temperature $\left(\mathrm{T}_{\mathrm{C}}\right)$ of $35 \mathrm{~K}$ [14]. This sparked up huge research work because of technological innovations which would emanate if the superconductor is made to operate at room temperature. At the end of 1986, Takagi et al confirmed the work of Georg Bednorz and Alex Müller by measuring the resistivity and diamagnetism of lanthanum barium copper oxide $(\mathrm{LaBa})_{2} \mathrm{CuO}_{4}$. In 1987, chemists in the Department of Magnetism of IP CSAS, led by E. Pollert discovered related compounds of cuprates-containing yttrium $\mathrm{YBa}_{2} \mathrm{Cu}_{3} \mathrm{O}_{7}$ with $\mathrm{T}_{\mathrm{C}}$ around $93 \mathrm{~K}$ [15]. The cuprates are type II superconductors. They have partial magnetic penetration which was explained by Abrikosov as mixed state.

Recently, the discovering of high-temperature superconductivity in iron-based compounds has sparked up another round of research. Yang et al.,[16] gave the similarities and differences between Fe pnictides and cuprates. Both are layered systems- having $d$ electrons playing a crucial role. The difference between the Fe pnictides and cuprates with respect to their d-electron count was given as six (even) and nine (odd) respectively [16]. An even number of d electrons implies that the parent state of their band-structure likely semiconductor while an odd number of the d electrons of the parent state of cuprates show that it can be modeled by half-filling band and band structure theories.

The cuprates systems have so far demonstrated maximum critical temperature range of $126 \mathrm{~K}-200 \mathrm{~K}$. Physicist, chemist and material scientist have in the past three decades made concerted efforts to improve on the critical temperature of cuprates to increase its temperature to room temperature. At room temperature, superconductors would employed for the conservation of world's power usage. In this paper, we reviewed the progress made so far on the cuprates family. We propounded a formular to theoretically validate if efforts on cuprates might possibly yield results in the nearest future.

The cuprates superconductors are thermodynamically unstable materials. Its mechanism remains volatile as new cuprates superconductors emerge. The mechanism of high Tc superconductivity was based on pairing i.e. non-retarded or retarded interactions [17]. This idea still remains controversial. Another prominent theories on the cuprates is the energy scales i.e. superconducting gap and the pseudo gap. This energy scales initiate the quasi-particle spectrum which had been proposed to explore the excitations of the superconducting cuprates. Its success was short lived because the reality of pseudo gap [18] is contested. Experimentalist and theorist's idea of phase coherence and fluctuations opened a new concept of superconductors in general. The cuprates was reported to possess much of phase coherence and fluctuations. Later, the critical point of a quantum phase transition [19] was introduced. The quantum phase transition (QPT) extends through few materials like heavy fermions and known magnetic materials. However, shortcomings were pronounced in cuprates which brought about the advanced quantum critical point (QCP) which is based on misfit strain between layers in a super-lattice [20]. The charge order in the misfit strain competes with the superconducting order parameter. The superconducting order parameter when viewed from the concept of magnetic pair breaking, hole localization, chemical internal pressure of individual cuprates component and cuprates disorder, showed consistent dependence on the critical temperature (Tc).

The hole localization i.e. doping in the QCP had been debated-upon both in over-doped and underdoped regions of the cuprates. Recently the universal dome-shaped behavior [21] was introduced to separate the over-doped and under-doped regions. Another popular concept of the critical temperature (Tc) of the cuprates superconductor is the layering of unit cells. This concept was found to be limited to layers below four. More recently, the idea of the self-assembled monolayer was introduced to solve the layering problem. This was achieved by organizing layer of molecules in which one end of the molecule and the binding group combines to form a monolayer. This method was found to improve the Tc of both over-doped and underdoped cupratesa. The proton irradiation technique was initially intended to introduce various defects into materials in a fairly predictable and controllable fashion. Undoubtedly, this idea has opened-up more atomic explanations into comprehending the cuprates superconductors. At low proton doses, there is a linear decrease in $T c$ and a linear increase of the extrapolated residual resistivity as proton dose is increased. This idea initiated other concepts like proton mobility in cuprates. Again, this idea was short lived since the highest Tc obtained for mercury cuprates was 95K [22].

Though most cuprates in recent times have shown a range of critical temperatures above $130 \mathrm{~K}$, there are still salient questions asked e.g. why does a decrease in the pairing gap result in a reduced critical temperature? How reliable is the chemical internal pressure to the critical temperature of the cuprates system? To what extent is the effect of layering cuprates related to the critical temperature? What mechanism is responsible for monotonic decrease in Tc when doping concentration is increased? How does a particulate 
repulsion of the Bose-Einstein condensate decrease or increase Tc? At what point does suppression of the superconducting critical temperature occur in cuprates? How related is the proton number to enhancing the critical temperature of cuprates superconductors?

Some of the answers are vague because the theories behind them are regarded as failed theories. Answers to other questions are summarized in a well articulated formula which is referred to as the Critical Temperature Model (CTM). Emetere [23]-[25] had given an in-depth insight of one the newest theories of superconductivity and how potentially useful they could become if utilized appropriately.

\section{RESEARCH METHOD}

After the emergence of the microscopic treatment of the convectional superconductors, salient truths about superconductors can be harvested from numerous studies for onward application. We shall examine three influential studies for this review. Gorkov [26] related the critical temperature and the gap in energy spectrum as

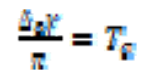

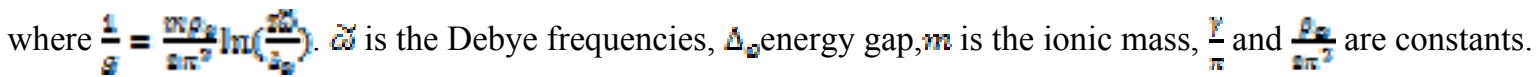
Furthermore, the relativistic framework of the microscopic theory of superconductivity establishes the atomic number concept for superconductors' band-structure calculations. Using the microscopic based Usadel theory, Martinis et al., [27] gave a relation of the transition temperature to some known constants i.e.

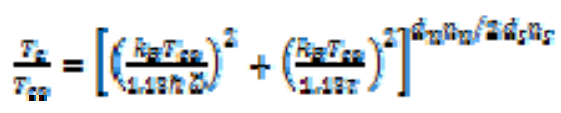

where $n_{n}$ and $n_{z}$ are the density of electronic states in the normal and superconducting films, $d_{n}$ and $u_{g}$ are the thickness of the normal and superconducting films, $F_{c o}$ the transition temperature of the bare superconductor, $k_{\vec{D}}$ is the Boltzmann constant, $\hbar$ is the Planck's constant, $\tau$ is the quantum conductivity ratio. Another microscopic treatment which relates the magnetic screening length of a two-dimensional hightemperature superconductor to its effective mass is given as

$$
\xi_{2}=\frac{m^{2} c^{2}}{2 \pi s_{g^{2}} e^{2}}
$$

$\xi_{s}$ is the magnetic screening length, $m^{*}$ is the effective mass of the superconducting carriers, $N_{s}$ is the sheet carrier density, $\theta$ is the elementary electronic charge and $\mathrm{c}$ is the speed of light.

Assume the following conditions under the sets of equations highlighted above

1. The sample is only a superconducting film and not a bare superconductor

2. Other unknown constants are captured only in magnitude.

3. The effective mass is related to atomic mass

4. The magnetic screening length is unity for a superconducting film.

Based on the aforementioned assumptions, the critical temperature model (CTM) for the cuprates was developed by Uno and Emetere [28] and its validity is discussed in the section below.

\section{ALGORITHM: VERIFYING CRITICAL TEMPERATURE MODEL}

Uno and Emetere [28] gave the critical temperature model as:

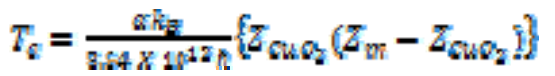

where $\mathrm{Z}_{\mathrm{CuO} 2}$ is the proton number of the copper-oxide site, $\mathrm{Z}_{\mathrm{m}}$ is the total proton number of the cuprates elemental constituents. $k_{\bar{q}}=$ Boltzmann constant given as $1.38 \times 10^{-23} \mathrm{JK}^{-\mathrm{I}}, \mathrm{h}=$ Planck's constant given as 6.26 $\mathrm{x} 10^{-34} \mathrm{Js}, \alpha=$ Active thermal proton mass participation which is given as $1 \mathrm{~K}^{2} \mathrm{~s}$.

The ionic mobility concept was applied to the CTM in equation (4) above. Though in convectional physics, temperature is inversely proportional to the mass of a body. On the contrary, in superconductivity, the reverse is the case experimentally [22] i.e. the temperature is almost linearly proportional to the mass of the cuprates superconductor. However, at suppressed superconductivity, the cuprates obeys the convectional 
physics. Again, the idea of Love et al.,[29] was employed where we viewed each layer of cuprates constituents as a layer of molecule $(\mathrm{Zm})$ and the $\mathrm{Cu}-\mathrm{O}$ plane as binding group $\left(\mathrm{ZCuO}_{2}\right)$. The mathematical arrangement i.e. $\mathrm{ZCuO}_{2}\left(\mathrm{Zm}-\mathrm{ZCuO}_{2}\right)$ suggests the square energy scale effect in superconductivity.

Hence, the reason for introducing the Boltzmann constant in Equation (1). The Boltzmann constant relates energy scales at the individual particle level with temperature observed at the collective or bulk cuprates layer. The Planck's constant is incorporated to quantum transformations within the layered cuprates. From the proton irradiance concept, the active thermal proton mass participation is incorporated to define the absorption of individual cuprates protons at certain temperature in the course of fabrication. Again, this concept (active thermal proton mass participation) is synonymous to the Bauschinger effect which refers to the effects of microscopic stress distribution as a result of characteristic changes in the material's stress/strain relationship [30]. In our numerical analysis, we assumed that the cuprates samples were homogenous i.e. $\alpha$ should be in the range of $1-2 \mathrm{~K}^{2} \mathrm{~s}$.

Application of the CTM was verified using experimental values of critical temperature of cuprates reported by researchers even though some inconsistencies in the data were noted. For example, Narkilar [31] reported the critical temperatures for Bismuth; Bi2201, Bi2212 and Bi2223 as 34K, 90K and $110 \mathrm{~K}$ respectively. Vlad [32] gave his value as $20 \mathrm{~K}$, $85 \mathrm{~K}$ and $110 \mathrm{~K}$ for Bi2201, Bi2212 and Bi2223 respectively. The values for T12201 were given by Narkilar [31], Raymond [33] and Vlad [32] as 90K, 95K and 85K respectively. Therefore the error boundary adopted using Narkilar's value was given as $\pm 5 \mathrm{~K}$. The summary of the verification of the CTM can be seen in Table 1 below.

Table 1. Verification of the CTM via Experimental Results

\begin{tabular}{lcccc}
\hline $\mathrm{S} / \mathrm{N}$ & CUPRATES & $\begin{array}{c}\text { EXPERIMENTAL } \\
\text { READING }\end{array}$ & $\begin{array}{c}\text { THEORETICAL } \\
\text { READING }\end{array}$ & $\boldsymbol{\Gamma}\left(\mathrm{K}^{2} \mathrm{~s}\right)$ \\
\hline 1. & $\mathrm{~T} 12201$ & $90 \pm 5 \mathrm{~K}[31-33]$ & $87.5 \mathrm{~K}$ & 1.00 \\
2. & $\mathrm{~T} 2212$ & $111.0 \pm 5 \mathrm{~K}[31-33]$ & $111.7 \mathrm{~K}$ & 1.00 \\
3. & $\mathrm{~T} 2223$ & $128.0 \pm 5 \mathrm{~K}[31-33]$ & $135.7 \mathrm{~K}$ & 1.00 \\
4. & $\mathrm{Cu} 2234$ & $113.0 \pm 5 \mathrm{~K}[31-33]$ & $114.7 \mathrm{~K}$ & 1.00 \\
5. & $\mathrm{~Pb} 1212$ & $70 \pm 5 \mathrm{~K}[31-33]$ & $71.1 \mathrm{~K}$ & 1.00 \\
6. & $\mathrm{~T} 11212$ & $82 \pm 5 \mathrm{~K}[31-33]$ & $81.5 \mathrm{~K}$ & 1.00 \\
7. & $\mathrm{Ru} 1212$ & $72 \pm 5 \mathrm{~K}[31-33]$ & $68.5 \mathrm{~K}$ & 1.00 \\
8. & $\mathrm{Au} 1212$ & $82 \pm 5 \mathrm{~K}[31-33]$ & $80.9 \mathrm{~K}$ & 1.00 \\
9. & $\mathrm{Bi2212}$ & $90 \pm 5 \mathrm{~K}[31-33]$ & $93.3 \mathrm{~K}$ & 1.00 \\
10. & $\mathrm{Bi} 2223$ & $110 \pm 5 \mathrm{~K}[31-33]$ & $112.1 \mathrm{~K}$ & 1.00 \\
11. & $\mathrm{YSr}_{2} \mathrm{Cu}_{3} \mathrm{O}_{7}$ & $62 \pm 5 \mathrm{~K}[31-33]$ & $61.5 \mathrm{~K}$ & 1.00 \\
12. & $\mathrm{Hg} 1201$ & $97 \pm 5 \mathrm{~K}[31-33]$ & $95.6 \mathrm{~K}$ & 1.53 \\
13. & $\mathrm{Hg} 1212$ & $128 \pm 5 \mathrm{~K}[31-33]$ & $123.6 \mathrm{~K}$ & 1.52 \\
\hline
\end{tabular}

\section{RESULTS AND ANALYSIS}

The costs of raw materials for cuprates production affect the low yield of the manufacturing process which has caused a significant drop in the recent applications of the cuprates. However, Yunhua et al, [34] describes a simple but reliable recycling process for failed bulk samples of the single grain high temperature superconductors where 64 samples were recycled with about $90 \%$ recovery. The key innovation of this technology is the process of re-growing failed samples from their solid states without necessarily regrinding the samples to powder form. Superconductivity of the recycled blend were measured and examined in order to ascertain their viability and it was discovered that the superconducting transition temperature Tc and the critical current density of small portions cut from the recycled grains showed similar characteristics with those of primary grown grains.

According to reports of the Basic Energy Sciences Workshop on superconductivity [35], following the discovery of high temperature (HTS) superconductors, fabrication of HTS materials a broad understanding of their properties and applications became the bane of research. These materials have been sought for application by various technologies but the strongest driver is the electric power sector. Power transmission through HTS cables offers chances of power recovery of some power that may be lost in the grid during transmission; also, the capacity of the grid is enhanced. HTS conductors also have the potential of improving the efficiencies, capacities and reliabilities of high-current carriers (devices). The first generation (1G) HTS conductors take the form Bi2Sr2Ca2Cu3O14 (BSCCO) which makes available conductors suitable for engineering tasks and first level applications in real power systems. The second generation (2G) HTS conductors have their origin in the following combination $\mathrm{YBa} 2 \mathrm{Cu} 3 \mathrm{O} 7$ (YBCO). The 2G HTS is an improvement on the $1^{\text {st }}$ generation HTS by virtue of its performance and reduced cost.

HTS can influence a nation or society's economy by providing the power utilities with sufficient capacity in the electric power grid system; this can be done by initiation of deregulation of utilities through 
its energy policies. Also in the area of information and communication engineering, high capacity fiber optic networks have transformed the economy by the availability of HTS which provides an almost unlimited capacity to transfer information across the globe. The power generation in a nation has the capacity to increase its overall GDP; for example, the growth rate demand for electricity in the US was about $2.3 \%$ per year which tracked closely with the country's GDP in 2006. Also, electricity is fast growing to become the most essential commodity form of energy which requires adequate market for it to be sustained. Going by the revolution that took place two decades ago a family of superconductors that have their root in ceramic oxides were discovered. They can withstand high temperatures and have the following formation- $\mathrm{HgBa} 2 \mathrm{Ca} 2 \mathrm{Cu} 3 \mathrm{O} 8$ temperatures as high as $164 \mathrm{~K}\left(-109^{\circ} \mathrm{C}\right)$ can be endured by such conductors. This makes cuprates a good candidate for communication [36],[37]. The crystal structure of this oxide allows for electrical signal to travel easily through certain crystal planes. Although, grand challenges still include the discovery of a roomtemperature superconductor and being able to unravel the mystery behind its mechanism.

Super conducting transformers work on the principle of superconductivity. They convert generator level voltage to high transmission level voltages which cuts down on the lost energy transmitted over long distances. Small, light-weight and efficient HTS transformers mounted at electrical substations can be used at utility grids to recover and redistribute this recovered energy. The beauty of this is that they can be sited in high density urban centers or buildings where conventional transformers could not be mounted. Also, unlike superconducting transformers significant energy losses are inherent in conventional transformers that possess an iron core where there are no-load losses and the copper in the windings where significant load losses occur.

High Temperature Superconductors (HTSs) are used as Fault Current Limiters (FCLs) in the power industry and are designed for use by electrical engineers in response to and to absorb unanticipated disturbances in a utility grid thereby mitigating power loss or damage to utility grid equipment. Their installation for electric utilities / high energy consumers in transmission and distribution systems is necessary because of the increased safety, the enhanced reliability, higher power quality, compatibility with other protection devices and the improved system flexibility due to the adjustable maximum allowable current and reduced capital investment for deferred upgrades. FCL super conductors instantaneously have the ability of limiting excess current flow by allowing itself to exceed its superconducting transition temperature i.e. (its critical temperature) and switch to a purely resistive or a seemingly passive state, thus minimizing the fault current passing through it.

HTS also find application in the operation of Reactive Power Generators (RGPs). The principle of operation of these devices is like that of an electric motor or generator without any connection to a real power source. Its duty is to supply or trap the reactive power needed to keep the flowing current and voltage in phase. The 8-MVAR machine manufactured by American Superconductor Corporation is an example of RGP that has been tested on Tennessee Valley Authority grid. Furthermore, the RGP is the the first commercial power equipment connected on HTS wire. There are anticipations that in the near future, these devices may be used in ensuring power stability. To buttress this point, in carrying out a close examination of the North America blackout scenario of 2003, U.S-Canada Task Force identified lack of RGPs as the principal cause of the cascading power outage. As a solution measure, it was noted that as the grid got more complex, in order to accommodate the increased energy demand for more generators, there was need to make provision for smart reactive power devices instead which will lead to a substantial purchase of renewable energy options such as solar energy, wind energy, and wave power that would incorporate additional instabilities in the grid system.

Another application of HTS is in refrigeration - thermodynamics (mechanical engineering). Liquid helium being a low temperature superconductor was a major drawback I early technology of refrigeration systems. Another challenge was the problem of thermally isolating its cryogenic windings. However, the advent of HTS, is a major breakthrough in this area because of this pronounced advantage over low temperature superconductors. Also, they eliminate the need for regular supply of liquid cryogens; although, the cooling capacity is not as efficient as it should be.

HTS can be seen as viable materials that have potentials for making of explosive devices e.g. plutonium, a radioactive metal that forms the explosive core of most nuclear weapons provides a link between heavy electrons and HTSs because of its ability to withstand high temperatures. However, the complex metallurgy of plutonium originates from the same electronic push-pull effect that exists between magnetism and electrical conductivity which subsequently gives rise to high temperature and heavy-electron superconductivity. These materials experience force competition where one of the two forces seem to prevail over the other i.e. at low temperatures, localization of electrons exist with a pull of electrons around a magnetic field which dominates the existing electrical force. However, at higher temperatures, electric forces dominate and delocalization of electrons take place as a result of the scattering electrons. The explosive core of plutonium has the ability to scatter off light as radiative source. 
Another area of application of HTS includes Materials and chemical engineering where refractory materials are used for high temperature applications. These HTS can be seen as composites that help to abate any unwanted stress distribution that may arise from high temperature disturbances in heaters used in the oil and gas industries for oil refining purposes. However, such temperature distributions along the composite materials can be handled due to the compact nature or structures formed by the blends inherent in HTSs. Again, in selecting some superconductive materials for applications, a good knowledge of material selection is necessary for the intended design applications. Problems such as stress distribution and fiber breakage can be avoided if their critical temperatures are known [38]

\section{CONCLUSION}

How reliable is the cuprates system? The cuprates could achieve the room temperature superconductivity but for its complex stoichiometry. Could doping cuprates actually achieve the room temperature superconductivity? Little success has actually been achieved. The dependence of the critical temperature on the proton mass participation shows within mathematical limits the shortcoming of the cuprates system. However, the relevance of the cuprates lies in the hybridize cuprates especially the $\mathrm{Cu}-\mathrm{O}$ plane, for example, hybridized copper $3 \mathrm{~d}$ and oxygen $2 \mathrm{p}$ orbital's. Considerable emphasis should also be drawn to the microscopic stress distribution of the hybrized cuprates monolayer as fabrication is essential for cuprates performance. Also, research needs to be carried out for a thorough grasp and proper understanding of the mechanisms behind the existence of a cuprates (high temperature super conductor) at room temperature as the right course to the desired outcome can only be charted by a better insight of the violation of the electron pairing principle that explains the mode of operation of conventional superconductors. Furthermore, an optimum point or point of compromise between the interactions of the magnetic field and electrical conductivity is required in order to attain the desired high temperature superconductor. Hence, there are numerous engineering applications of cuprates if its critical temperature could be optimized.

\section{ACKNOWLEDGEMENTS}

The authors appreciate the partial sponsorship of the Covenant University.

\section{REFERENCES}

[1] K. Onnes, "Communications from the Physical Laboratory of the University of Leiden," vol. 29, pp. 1, 1911.

[2] Thompson J. J., "Conduction of Electricity through Metals," Philosophical Magazine, vol. 30, pp. 192, 1915.

[3] E. Albert, "Theoretische Bemerkungen zur Supraleitung der Metalle," in Het Natuurkundig Laboratorium der Rijksuniversiteit te Leiden in de Jaren, pp. 429, 1922.

[4] Heisenberg W., "Zur Theorie des Ferromagnetismus," Zeitschift für Physik, vol. 49, pp. 619, 1928.

[5] Bloch F., "Quantenmechanik der Elektronen in Kristallgittern," Zeitschift für Physik, vol. 52, pp. 555, 1928.

[6] Bohm D., "Note on a Theorem of Bloch Concerning Possible Causes of Superconductivity Physical Review," vol. 75, pp. 502, 1949.

[7] Landau L. D., “Zur Theorie der Phasenumwandlungen I,” Physikalische Zeitschrift der Sowjetunion, vol. 4, pp. 675 1933.

[8] Landau L. D., "Statistical theory of nuclei," Soviet Physics JETP, vol. 7, pp. 19, 1937.

[9] Brillouin L., "Le modele d'atome de Fock-Dirac et I'existence des potentiels d'ionisation," J. phys. et rad., vol/issue: 7(5), pp. 185-192, 1934.

[10] London F., "On the problem of the molecular theory of superconductivity," Physical Review, vol. 74, pp. 562-573, 1948.

[11] Fröhlich H., "Theory of the Superconducting State I. The Ground State at the Absolute Zero of Temperature," Physical Review, vol. 79, pp. 845, 1950.

[12] J. Bardeen, et al., "Microscopic Theory of Superconductivity," Phys. Rev., vol. 106, pp. 162-164, 1957.

[13] M. R. Schafroth, "Remarks on the Meissner Effect," Phys. Rev., vol. 111, pp. 72-74, 1958.

[14] Bernorz J. G. and K. A Müller, "Low-field microwave absorption in the superconducting copper oxides," z. phys. B., vol. 64, pp. 189, 1986.

[15] Wu M. K., et al., "Superconductivity at $93 \mathrm{~K}$ in a new mixed-phase Y-Ba-Cu-O compound system at ambient pressure," phys.Rev.Lett, vol. 58,pp. 908, 1987.

[16] Yang W. L., et al., "Evidence for weak electronic correlations in iron pnictides," Phys. Rev. B., vol. 80, pp. 014508, 2009.

[17] Maier T. A., et al., "Dynamics of the Pairing Interaction in the Hubbard and $t-J$ Models of High-Temperature Superconductors," Phys. Rev.Lett., vol. 100, pp. 237001, 2008.

[18] Hufner S., et al., "Two gaps make a high-temperature superconductor?" Reports on Progress in Physics, vol/issue: 71(6), pp. 062501, 2008.

[19] Sachdev S., "Colloquium: Order and quantum phase transitions in the cuprates superconductors," Rev. Mod. Phys., vol. 75 , pp. $913,2003$. 
[20] Bianconi A., et al., "A quantum phase transition driven by the electron lattice interaction gives high TC superconductivity," J. Alloys Compd., vol. 317, pp. 537, 2001.

[21] G. Buzon, et al., "Self-Energy Effects In Cuprates and the Dome-Shaped Behavior of the Superconducting Critical Temperature," Phys. Rev. B., vol. 89, pp. 024516, 2014.

[22] Y. M. Baikov, et al., "Superconductivity and high proton mobility in hydrogen-containing cuprates of the 123 family with an elevated oxygen concentration," Tech. Phys. Lett., vol. 209, pp. 696, 1994.

[23] M. E. Emetere, "Presence of Pseudo-Path in the Inter-Plane Penetration Depth of Layered YBa2Cu3Oy," Journal of Superconductivity and Novel Magnetism, vol/issue: 28(4), 2015. DOI 10.1007/s10948-014-2939-8.

[24] M. E. Emetere, "Effects of Tunable Bloch Inspired Spin Orbit Interaction in the Electronic State Of Sr2RuO4," Journal of Superconductivity and Novel Magnetism, vol/issue: 28(1), pp. 231-239, 2015. DOI 10.1007/s10948-0142848-x.

[25] M. E. Emetere, "Characteristic Significance of Magnetic Relaxations on Copper Oxide Thin Film Using the Bloch NMR," Surface Review and Letters, vol/issue: 21(5), pp. 1450075, 2014. DOI: 10.1142/S0218625X14500759.

[26] Gorkov L. P., "Microsopic Derivation of Ginzburg-Landau Equation in the Theory of Superconductivity," Soviet Physics JEPT, vol. 36, pp. 1365, 1959.

[27] J. M. Martinis, et al., "Calculation of $\mathrm{T}_{\mathrm{c}}$ in a normal-superconductor bilayer using the microscopic-based Usadel theory," Nuclear Instruments and Methods in Physics Research A., vol. 444, pp. 23-27, 2000.

[28] U. E. Uno and M. E. Emetere, "Isotope Effect on Cuprates Component in Determining Experimental Critical Temperature," Int. Journal for scientific research, vol/issue: 1(1), pp. 15-24, 2011.

[29] Love J. C., et al., "Self-Assembled Monolayers of Thiolates on Metals as a Form of Nanotechnology," Chem. Rev., vol. 105, pp. 1103-1169, 2005.

[30] S. Chénais, et al., "On thermal effects in solid state lasers: the case of ytterbium-doped materials," Progress in quantum electronics, vol. 30, pp. 89-126, 2006.

[31] V. Grigore, et al., "Correlational Analysis Of Layered Superconducting Cuprates," WSEAS Transaction on Information Science \& Applications, vol/issue: 5(7), pp. 1180-1188, 2008.

[32] Narlikar A. V., "High Temperature Superconductivity 2: Engineering Applications 1 Materials," Springer, Berlin, 2004.

[33] R. A. Serway and J. W. Jewett, "Physics for Scientists and Engineers: Chapters 12 (7th edition)," ISBN9780495013129, pp. 123, 2005.

[34] S. Yunhua, et al., "A Reliable Method of Recycling (RE)-Ba-Cu-O (RE: Sm, Gd, Y) Bulk Superconductors," Journal of American Ceramic Society, vol/issue: 98(9), pp. 2760-2766, 2015.

[35] "Report of the Basic Energy Sciences Workshop on Superconductivity Office of Science," US Department of Energy, Argonne National Laboratory, pp. 1-43, 2011. http://www.sc.doe.gov/bes/reports/files/SC_rpt.pdf.

[36] M. E. Emetere, "Theoretical Modeling of a Magnetic Loop Antenna for Ultra wideband (UWB) Application," TELKOMNIKA Indonesian Journal of Electrical Engineering, vol/issue: 12(10), pp. 7076 - 7081, 2014.

[37] M. E. Emetere, "Magnetically Controlled Quantum Teleportation of Multiple Arbitrary States Using the Bloch Catalyst," TELKOMNIKA Indonesian Journal of Electrical Engineering, vol/issue: 13(1), pp. 1-9, 2015.

[38] S. M. Brian, "An Introduction to Materials Engineering and Science for Chemical and Material Engineers," John Wiley and Sons, Pp. 681-795, 2004. 